\title{
Tunable reflection minima of nanostructured antireflective surfaces
}

\author{
S. A. Boden a) and D. M. Bagnall \\ Electronics and Computer Science, University of Southampton, Highfield, Southampton, Hampshire \\ SO17 1BJ, United Kingdom
}

(Received 7 August 2008; accepted 10 September 2008; published online 30 September 2008)

\begin{abstract}
Broadband antireflection schemes for silicon surfaces based on the moth-eye principle and comprising arrays of subwavelength-scale pillars are applicable to solar cells, photodetectors, and stealth technologies and can exhibit very low reflectances. We show that rigorous coupled wave analysis can be used to accurately model the intricate reflectance behavior of these surfaces and so can be used to explore the effects of variations in pillar height, period, and shape. Low reflectance regions are identified, the extent of which are determined by the shape of the pillars. The wavelengths over which these low reflectance regions operate can be shifted by altering the period of the array. Thus the subtle features of the reflectance spectrum of a moth-eye array can be tailored for optimum performance for the input spectrum of a specific application. () 2008 American Institute of Physics. [DOI: 10.1063/1.2993231]
\end{abstract}

Reduction of optical reflection is important for many technologies including solar energy, photodetectors, and high-contrast, antiglare, and stealth surfaces. Traditionally the approach has been to exploit destructive interference by application of single-layer antireflective coatings but these can only reduce reflection to very low levels for the specific wavelength for which they are designed. ${ }^{1}$ More broadband antireflection (AR) can be achieved with double or triple layer coatings but the availability of materials with appropriate refractive indices and problems with the matching of thermal expansion coefficients limit the applicability of these AR schemes. For very low reflection, thin film coatings are often combined with texturing at dimensions greater than the wavelength of light. An example of this, the inverted pyramid scheme found on high efficiency solar cells, ${ }^{2,3}$ is a highly effective scheme but is incompatible with thin devices.

Taking inspiration from the eyes and wings of some species of moth ${ }^{4-6}$ [Fig. 1(a)], quartz ${ }^{7,8}$ polymer, ${ }^{9} \mathrm{GaSb},{ }^{10}$ and silicon ${ }^{11-15}$ have been patterned on a scale below the wavelength of incident light to create biomimetic "moth-eye" AR surfaces. A simple understanding of the AR process can be gained by considering incident light responding to a spatial average of the optical properties of any given interface volume dependent on the fraction of substrate to surrounding medium. The features on moth-eye surfaces are tapered and so this fraction gradually increases from the surrounding medium to the substrate, effectively blurring the interface and thereby reducing reflection across a relatively broad spectral range with reflection increasing at long wavelengths (when the interface thickness is small compared to the wavelength) and at short wavelengths (when structures are no longer subwavelength and are diffractive).

In this paper, we use simulation and experimental studies to illustrate how this simple effective medium approach cannot accurately predict important features of moth-eye reflection spectra. We show that because the scale of moth-eye features is often only just below the wavelength of incident light, the height, shape, and interpillar spacing (period) of

${ }^{a)}$ Electronic mail: sb1@ecs.soton.ac.uk. designs can have a pronounced effect on optical properties and must therefore be considered for specific applications.

Electron beam lithography is used to define regular arrays of circles in a $110 \mathrm{~nm}$ layer of FOx-12 flowable oxide (Dow Corning) on a silicon wafer. The period (pillar-to-pillar distance) of the pattern is varied from 150 to $350 \mathrm{~nm}$, and the duty cycle (pillar width to period ratio) is varied from $\sim 0.3$ to $\sim 0.7$. Each pattern is limited to a $1 \mathrm{~mm}^{2}$ square to allow reasonable write times. An $\mathrm{HBr}$ anisotropic dry etch transfers the pattern into the underlying silicon to create pillars with heights varying from $\sim 150$ to $\sim 500 \mathrm{~nm}$. An example of the resulting structures is shown in Fig. 1(b). For some samples, a $20 \mathrm{~nm}$ thermal oxide was grown and then removed to further shape the pillars.

Reflectance at near-normal incidence is measured using a reflectance probe with a tungsten-halogen white light source and a spectrometer. Polished silicon is used as a reflectance standard and the theoretical reflectance spectrum for silicon is used to calculate the absolute reflectance of silicon moth-eye samples.

AR surfaces are modeled using rigorous coupled wave analysis (RCWA) (Ref. 16) implemented using the commercial software package GD-CALC. ${ }^{17}$ A typical moth-eye array is approximated by a stack of cylinders (which themselves are approximated by an arrangement of cuboids) with increasing radii from air to the substrate. A cosine-based function is used to describe the variation of radius with height to produce a biomimetic pillar shape. The reflectance is obtained

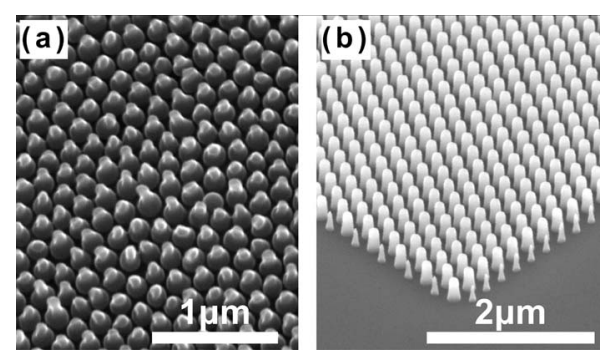

FIG. 1. SEM images of (a) antireflective subwavelength features found on the transparent section of the wing of the Cryptotympana Aquila. (b) A biomimetic silicon moth-eye surface. 

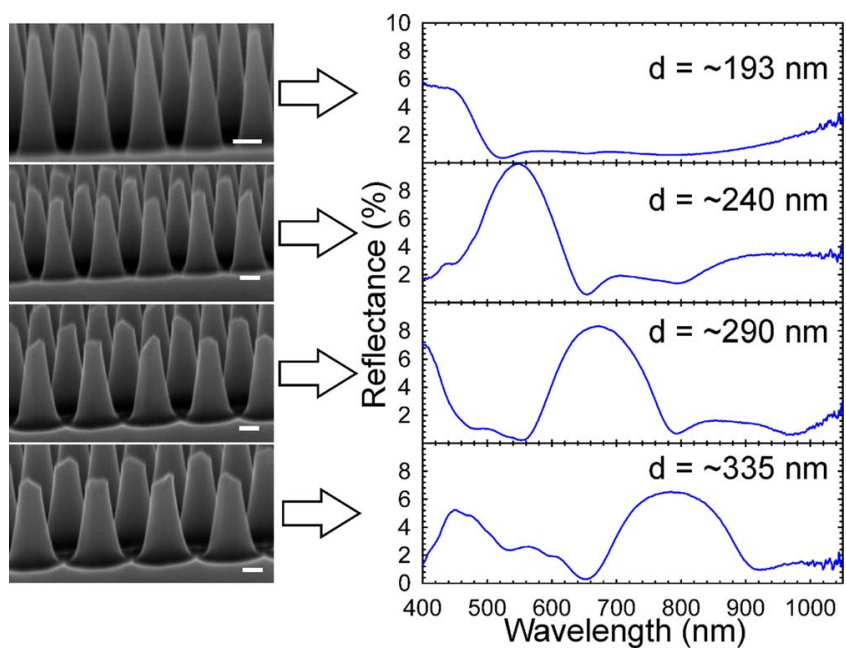

FIG. 2. (Color online) Measured reflectance spectra for silicon moth-eye surfaces of different periods. The scale bars in the SEM images are all $100 \mathrm{~nm}$.

by summing the reflected order efficiencies from the RCWA calculations.

Results from our experiments (Fig. 2) show that a silicon moth-eye surface can exhibit extremely low reflectances (below $1 \%$ for wavelengths from 504 to $834 \mathrm{~nm}$ for a period of $\sim 193 \mathrm{~nm})$. However, the reflectance spectra exhibit a series of local maxima with spectral positions strongly dependent on the period of the array. Our simulations, based on similar nanostructure designs [Figs. 3(a) and 3(b)]. show similar spectral features and dependencies.

These experimental and simulation results have important implications for AR design. The periodicity (and indeed shape and height) of the moth-eye design must be carefully considered. There will, for instance, be an optimal periodicity for solar cells, based on a superposition of AR spectra, the solar spectra, and the internal quantum efficiency of the device. In the case of AR surfaces for laser light, we can also see that careful design could provide reflectance as low as $0.1 \%$. With this in mind, we have performed a number of simulations to provide further insight into the properties of these surfaces.

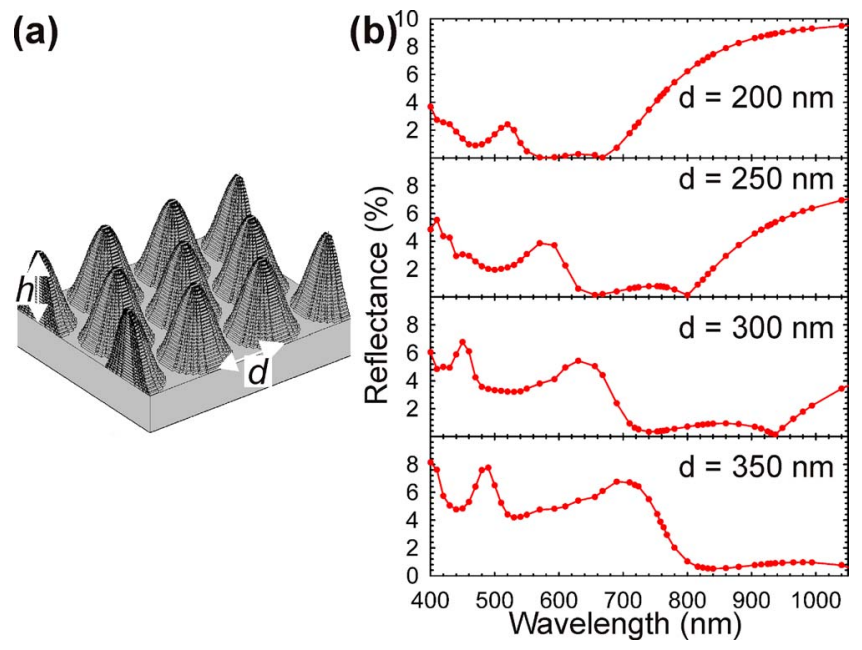

FIG. 3. (Color online) (a) Biomimetic moth-eye profile defined in simulation software. (b) Simulated reflectance spectra for silicon moth-eye arrays with a pillar height of $400 \mathrm{~nm}$ and various periods.

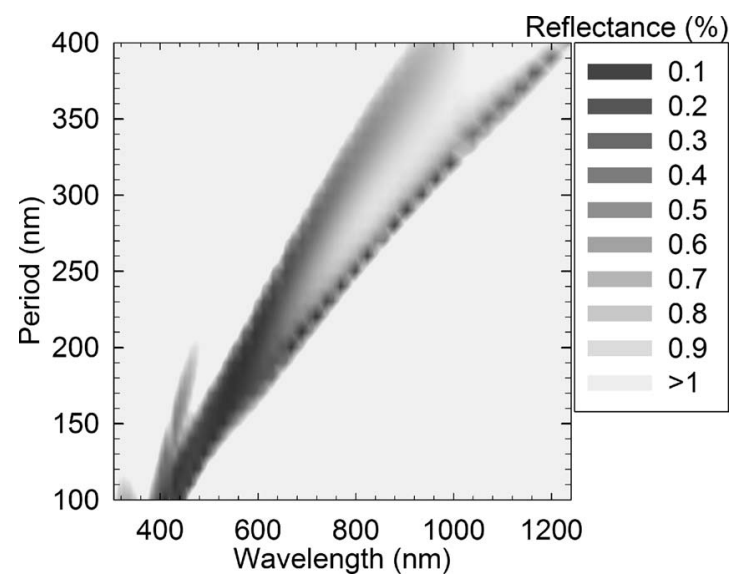

FIG. 4. Simulated parameter map of the variation of reflectance with period and wavelength for the same silicon moth-eye array shown in Fig. 3(a). Only reflectances below $1 \%$ are plotted.

Reflectance calculated for a range of wavelengths and periods are presented as a contour plot in Fig. 4. Only reflectances below $1 \%$ are plotted to highlight the low reflectance band, which broadens and shifts to higher wavelengths as the period is increased and is split at higher periods into two minima.

Further simulations were conducted to analyze the effects of changing pillar height. Calculations of reflectance were performed for different pillar heights and periods at a wavelength $\lambda$ of $1000 \mathrm{~nm}$ (Fig. 5). For small periods (i.e., $d \ll \lambda$ ), the array of tapered pillars exhibits properties similar to those that would be expected for an interface with a graded refractive index. ${ }^{18,19}$ At small pillar heights, the reflectance is high as the interface appears abrupt to incoming light. As the height is increased, there is a sharp drop in reflectance, which stays low but with some low amplitude periodic variations. These are attributed to interference due to discontinuities in the refractive index profile at the boundaries of the graded index layer. For periods approaching the wavelength, more complicated reflectance behavior due to resonance effects is observed. In between these regimes, we

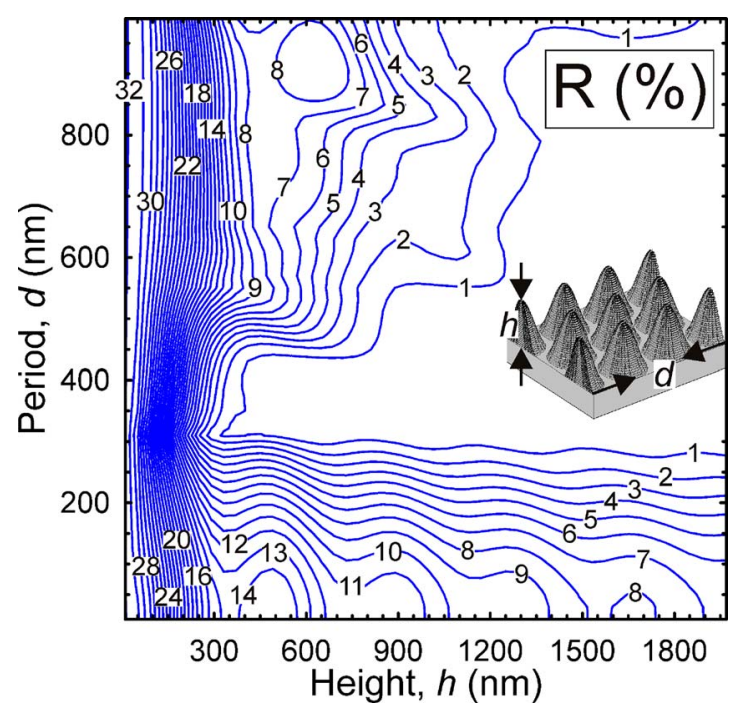

FIG. 5. (Color online) Parameter scan of reflectance of light with a wavelength of $1000 \mathrm{~nm}$ vs height and period for a hexagonal array of pillars with the biomimetic moth-eye profile (inset). The silicon refractive index used for a wavelength of $1000 \mathrm{~nm}$ is $3.617+0.0041 i$ (Ref. 20). 
(a)
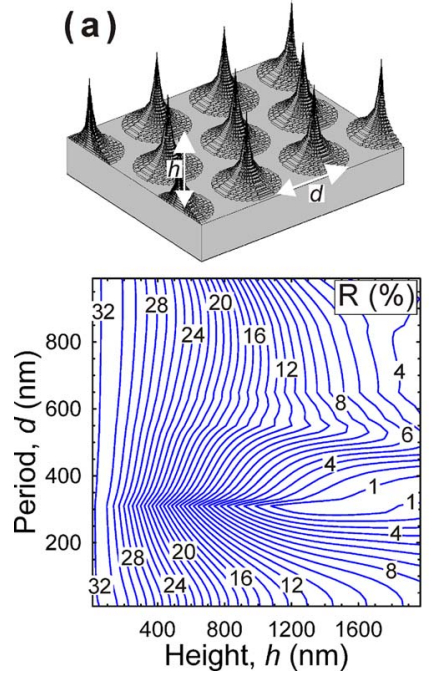

(b)
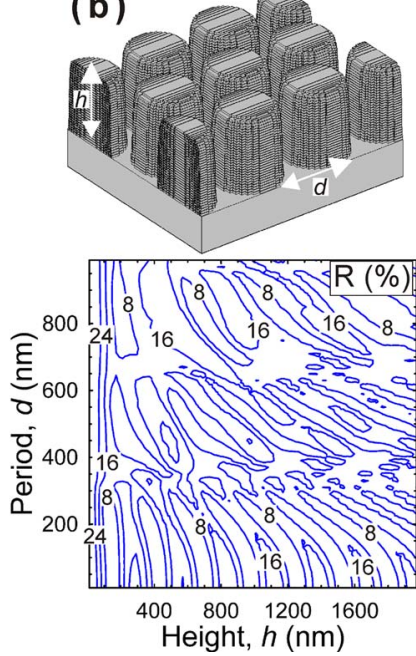

FIG. 6. (Color online) Parameter scan of reflectance of light with a wavelength of $1000 \mathrm{~nm}$ vs height and period for a hexagonal array of pillars with the pillar shapes shown. The silicon refractive index used for a wavelength of $1000 \mathrm{~nm}$ is $3.617+0.0041 i$ (Ref. 20).

find a valley in which a low reflectance band appears at relatively modest pillar heights. This represents a "sweet spot" for the design of moth-eye surfaces because shorter pillars are easier to fabricate.

In general, the taller the pillars, the lower the reflectance; however, in the case of moths or solar cells, it is clear that very tall features or large surface areas would be costly. Given such constraints, it is generally a case of diminishing returns for pillar heights beyond around half of the wavelength.

Changing the shape of the moth-eye array pillars also has a dramatic effect on the reflectance behavior. The reflectance versus height and period contour plot for a moth-eye array with sharper pillars [Fig. 6(a)] also exhibits a low reflectance region; however, this extends over a smaller period range than that of the biomimetic moth-eye profile shown in Fig. 5. This would lead to a sharper minimum in the reflectance spectrum and so a surface that is less suitable for broadband applications. There is also a more gradual decrease in reflectance with height and so taller pillars are required to reach low reflectance.

At the other extreme, pillars with near-vertical side walls and flat tops [Fig. 6(b)] exhibit rapid variations in reflectance with height and period. At small periods, this can be attributed to the array acting as an effective medium that approximates a single layer thin film. Interference of reflections from the top and bottom of the layer causes the maxima and minima seen as pillar height is increased. This design is far from ideal for broadband applications because of the sensitivity of antireflective performance to small changes in parameters. The biomimetic design (Fig. 4) exhibits properties closer to an optimum than the two other designs investigated here.

It is important to note that many of these effects including the shifting with period of a low reflectance region are not predicted with effective medium approaches. Indeed, the variation in reflectance spectra with period cannot be analyzed using an effective medium theory because this approach assumes homogenous in-plane properties. It is therefore necessary to use rigorous solutions to Maxwell's equations to accurately predict and therefore tailor the properties of moth-eye surfaces with subwavelength-scale features.

In conclusion, our studies indicate that in order to achieve the highest performance from a moth-eye antireflective surface for a specific application the pillar height, shape, and array period should be optimized for the specific wavelength range over which the surface is required to operate. In this regard, we have found simulations by RCWA to be able to provide a strong indication of how these parameters affect the reflectance and thereby provide appropriate designs for surfaces optimized for applications.

${ }^{1}$ J. Zhao and M. A. Green, IEEE Trans. Electron Devices 38, 1925 (1991). ${ }^{2}$ P. Papet, O. Nichiporuk, A. Kaminski, Y. Rozier, J. Kraiem, J. F. Lelievre, A. Chaumartin, A. Fave, and M. Lemiti, Sol. Energy Mater. Sol. Cells 90, 2319 (2006).

${ }^{3}$ J. Zhao, A. Wang, M. A. Green, and F. Ferrazza, Appl. Phys. Lett. 73, 1991 (1998).

${ }^{4}$ C. G. Bernhard, Endeavour 26, 79 (1967).

${ }^{5}$ A. R. Parker, J. Opt. A, Pure Appl. Opt. 2, R15 (2000).

${ }^{6}$ P. Vukusic and J. R. Sambles, Nature (London) 424, 852 (2003).

${ }^{7}$ R. C. Enger and S. K. Case, Appl. Opt. 22, 3220 (1983).

${ }^{8}$ H. Toyota, K. Takahara, M. Okano, T. Yotsuya, and H. Kikuta, Jpn. J. Appl. Phys., Part 2 40, L747 (2001).

${ }^{9}$ P. B. Clapham and M. C. Hutley, Nature (London) 244, 281 (1973).

${ }^{10}$ W. L. Min, A. P. Betancourt, P. Jiang, and B. Jiang, Appl. Phys. Lett. 92, 141109 (2008).

${ }^{11}$ Y. Kanamori, M. Sasaki, and K. Hane, Opt. Lett. 24, 1422 (1999).

${ }^{12}$ P. Lalanne and G. M. Morris, Nanotechnology 8, 53 (1997).

${ }^{13}$ Y. F. Huang, S. Chattopadhyay, Y. J. Jen, C. Y. Peng, T. A. Liu, Y. K. Hsu, C. L. Pan, H. C. Lo, C. H. Hsu, Y. H. Chang, C. S. Lee, K. H. Chen, and L. C. Chen, Nat. Nanotechnol. 2, 770 (2007).

${ }^{14}$ S. A. Boden and D. M. Bagnall, Proceedings of the Third Photovoltaic Science Applications and Technology Conference (PVSAT-3), Durham, UK, 2007 (unpublished).

${ }^{15}$ C.-H. Sun, P. Jiang, and B. Jiang, Appl. Phys. Lett. 92, 061112 (2008).

${ }^{16} \mathrm{M}$. Nevière and E. Popov, Light Propagation in Peirodic Media (Dekker, New York, 2003).

${ }^{17}$ K. C. Johnson, GD-Calc, 2005.

${ }^{18}$ W. H. Lowdermilk and D. Milam, Appl. Phys. Lett. 36, 891 (1980).

${ }^{19}$ S. J. Wilson and M. C. Hutley, Opt. Acta 29, 993 (1982).

${ }^{20}$ S. Adachi, Phys. Rev. B 38, 12966 (1988). 